\title{
Continuous glucose monitoring devices: A brief presentation (Review)
}

\author{
DOINA ANDRADA MIHAI ${ }^{1,2}$, DIANA SIMONA STEFAN ${ }^{1,2}$, DANIELA STEGARU $^{1,2}$, \\ GEORGIANA ELENA BERNEA ${ }^{2}$, ILEANA ADELA VACAROIU ${ }^{3,4}$, TOMA PAPACOCEA ${ }^{5}$, \\ MIRCEA OVIDIU DENIS LUPUȘORU ${ }^{6}$, ADRIANA ELENA NICA ${ }^{7}$, OVIDIU STIRU $^{8,9}$, \\ DORIN DRAGOS $^{10,11}$ and OCTAVIAN GABRIEL OLARU ${ }^{12}$
}

\begin{abstract}
${ }^{1}$ Discipline of Diabetes, Nutrition and Metabolic Diseases, Faculty of Medicine, 'Carol Davila' University of Medicine and Pharmacy, 'N. C. Paulescu' Institute of Diabetes, Nutrition and Metabolic Diseases, 020021 Bucharest;

${ }^{2}$ 'N. C. Paulescu' Institute of Diabetes, Nutrition and Metabolic Diseases, Diabetes Department II, 020474 Bucharest; ${ }^{3}$ Department of Nephrology, Faculty of Medicine, 'Carol Davila' University of Medicine and Pharmacy, 020021 Bucharest;

${ }^{4}$ Department of Nephrology and Dialysis, 'Sf. Ioan' Emergency Clinical Hospital, 042122 Bucharest;

${ }^{5}$ Department of Neurosurgery and ${ }^{6}$ Discipline of Physiology, Faculty of Medicine, 'Carol Davila' University of

Medicine and Pharmacy; ${ }^{7}$ Department of Orthopedics, Anesthesia Intensive Care Unit and ${ }^{8}$ Department of

Cardiovascular Surgery, Faculty of Medicine, 'Carol Davila' University of Medicine and Pharmacy, 020021 Bucharest;

${ }^{9}$ Department of Cardiovascular Surgery, 'Prof. Dr. C.C. Iliescu' Emergency Institute for Cardiovascular Diseases, 022322 Bucharest; ${ }^{10}$ Department of Medical Semiology, Discipline of Internal Medicine I and Nephrology,

Faculty of Medicine, 'Carol Davila' University of Medicine and Pharmacy, 020021 Bucharest;

${ }^{11}$ Department of Internal Medicine I, Emergency University Hospital, 050098 Bucharest; ${ }^{12}$ Department of Obstetrics and Gynecology, Faculty of Medicine, 'Carol Davila' University of Medicine and Pharmacy, 020021 Bucharest, Romania
\end{abstract}

Received October 6, 2021; Accepted November 5, 2021

DOI: $10.3892 / \mathrm{etm} .2021 .11097$

\begin{abstract}
As diabetes prevalence is continuously increasing, better management is needed to achieve blood glucose control, in order to prevent complications and lessen the burden of this disease. Since the first measurement of glycosuria at the beginning of the 1900s', huge advances were made in monitoring glycemia. Continuous glucose monitoring systems revolutionized diabetes management, especially for patients with type 1 diabetes. Avoiding glycemic variability and maintaining optimal glycemic control is crucial for the evolution of patients with type 1 diabetes. The usefulness of glycemic monitoring devices can be extended to patients with type 2 diabetes. It is also important to note that in those patients at risk of developing high glycemic variability (e.g. patients with advanced chronic kidney disease), continuous glycemic monitoring may improve their prognosis. These monitoring systems can be classified according to the analytical method, the degree
\end{abstract}

Correspondence to: Dr Ileana Adela Vacaroiu, Department of Nephrology, Faculty of Medicine, 'Carol Davila' University of Medicine and Pharmacy, 12 Dionisie Lupu Street, 020021 Bucharest, Romania E-mail: ileanaadela@yahoo.com

Key words: continuous glucose monitoring systems, glucose detection technologies, diabetes, biosensors, polymers of invasiveness, the data availability and the mode of usage. The technology is constantly improving in bioanalytical performance, biocompatibility, length of wearing time, safety and clinical features. The aim of this review was to briefly present the main characteristics of glucose biosensors, glucose monitoring systems and their clinically utility.

\section{Contents}

1. Introduction

2. Continuous glucose monitoring systems (CGMS): Characteristics

3. CGMS: In clinical practice

4. Conclusions

\section{Introduction}

Diabetes is rising worldwide with an estimated prevalence of 463 million affected people in 2019 and the numbers are expected to increase even more in the years to come, with an estimated figure of 578 million cases by 2030 (1). Due to the very high prevalence and the serious chronic complications, including cardiovascular, renal, ocular and neural complications $(2,3)$ and the frequent association with other diseases such as high blood pressure, dyslipidemia (4) or other cardiovascular risk factors (5), diabetes is a major health issue 
worldwide and a heavy burden on healthcare systems. In order to minimize this burden, good glycemic control is crucial, since the diagnosis, is the key to prevent chronic complications. Assessing blood glucose levels or other indicators of glycemic control such as glycosuria, glycated hemoglobin and glycated albumin (6) is therefore an essential tool in diabetes treatment. In the mid-1800s, the first attempts to quantify glucose were made and glucose was measured in urine, using evaporation of urine to obtain sugar crystals. In the first decade of the 1900s, a copper reagent was developed for assessing glucose in urine and tests for glycosuria started to be commercialized (7). The first blood glucose test strip that used glucose oxidase and a chromagen indicator system was available in Great Britain and then in the United States in 1964 under the name Dextrostix, although it was only accessible to the physician (8). At the beginning of the 1980 s, the first blood glucose meter was developed under the name Dextrometer and could be used by the patients at home (9). Since then, self-monitoring of blood glucose (SMBG) gradually became a standard in diabetes care. In the following decades, the technique of SMBG constantly improved and was characterized by a reduction in the amount of required blood $(<1 \mu \mathrm{l})$, an improved suction of blood sample by capillary action, more accurate results, fewer interferences (e.g. with hematocrit), the use of electrochemical strips, new enzymatic tests (glucose dehydrogenase), reduced meter size, better lancets, and a higher affordability for diabetic patients. The advances in SMBG techniques led to an improvement in diabetes control. However, some limitations exist. Even with multiple daily blood glucose measurements, the test only provides instantaneous information, the repeated finger pricks are inconvenient, and there cannot be any warnings about hypoglycemia or hyperglycemia.

\section{Continuous glucose monitoring systems (CGMS): Characteristics}

The development of CGMS was a real revolution in diabetes care because it provided the whole daily picture of blood glucose values. CGMS measures glucose levels in the interstitial fluid. The first in vivo needle-type continuous glucose sensor was developed by Shichiri et al (10) in 1982. The first CGM was approved by the Food and Drug Administration in 1999 (7). It was a retrospective system (blinded) intended for professional office use only. The patient had to wear the device for three days and data were collected by the physician for further analysis.

CGMS comprises the three following parts: A sensor that measures interstitial glucose levels, a receiver or monitor that displays data, and a transmitter that enables the communication between the other two components. Nowadays, CGMS can be used alone or in combination with an insulin pump. The sensor is inserted into the subcutaneous tissue by a small skin puncture. The level of glucose measured in the interstitial fluid is assumed to be similar to that measured in the capillary, although when glucose levels vary too fast such as during exercise or postabsorptive state, there are some discrepancies due to physiological delays in time (11). Sensors differ in many aspects, from assay method, invasiveness to clinical usage. Taking into account these characteristics, the systems can be classified in multiple manners.
First, CGMS can be classified by the analytical method used to quantify glucose, as extensively review by Nichols et al (12). There are three main technologies for glucose detection: Electrochemical (enzymatic and nonenzymatic), optical (fluorophore-based and nonflourophore based), and combinational or other types (piezoelectric, electromagnetic impedance) (12-14).

Biosensors used to monitor glucose levels have evolved over time, and three generations can now be identified (15). The first-generation sensors were based on the use of natural oxygen and hydrogen peroxide detection. The second-generation sensors focused on the non-physiological electron acceptor. The third generation of sensors stood out by modernizing the design with changing the artificial mediators and even in some cases giving up the enzyme system.

There is a tendency to switch from invasive sensors to non-invasive methods, to increase the life of the sensors from a maximum of 7 days to over a month or even years, and to provide safety against infections using specific coated materials (16-18).

Enzymatic electrodes are based on the action of enzymes that catalyze redox reactions by creating an electric current with measurable voltage that is dependent of glucose concentration. At present, numerous variants of invasive enzymatic biosensors have appeared with different technologies, including the following: Microdialysis, micropores, microneedles, subcutaneous amperometric electrodes and intravenous implantable devices, of which only those based on microdialysis and subcutaneous are commercially available (19).

The gold standard of the current biosensors in use is represented by the invasive Platinum-Iridium (Pt-Ir) needle-type ones. These needles are inserted subcutaneously to come in contact with the interstitial fluid and can detect glucose by enzymatic method. Classic needle type consists of a Pt-Ir wire with a diameter of $0.125 \mathrm{~mm}$, for $\mathrm{Pt}: \mathrm{Ir}=9: 1$, which represents the active electrode, having an immobilized mediator and, at the surface an enzyme, all covered by a polymer coating to facilitate subcutaneous implantation. Counter electrode is represented by a silver wire/silver chloride $(\mathrm{Ag} / \mathrm{AgCl})$ wrapped around the active electrode (19).

The microdialysis sensors are based on a hollow fiber that captures interstitial glucose (20). A microfluidic chip with microchannels in polydimethylsiloxane ensures an effective mixing of dissolved glucose oxidase and microdialysis sample in nanoliter volumes. The Pt electrodes are protected by a polypyrrole coating to prevent protein adsorption and interference with various substances such as uric acid, ascorbic acid or acetaminophen (20). The coating is overoxidized in order to became nonconductive and permselective (20). The microfluidic chip with an integrated chaotic mixer is combined with an enzymatic microreactor. The system proved good glucose sensibility in rodents (20).

Another advance in implantable sensors was realized by the development of coil-type oxidase-biosensors, based on the classic needle-type sensors (19). The Pt wire is wound up around a 30-gauge needle forming a coil-shaped cylinder and increasing the sensing area. The use of advanced techniques such as optical and electronic microscopy to assess the structure of biomaterial after the interaction with the human body may be useful for future new materials (21). 
In the last decade, the possibility of making new biosensors from materials such as carbon nanotubes (CNTs) and graphene has begun to be evaluated (22). CNT nanotubes can be used as electrical wiring/connectors with redox enzymes. CNTs look like an electric wire but have the advantage that the topography of the surface and porosity facilitate the action of enzymes (22-25).

Technological development is trying to transition from biosensors that are based on an enzymatic mechanism to enzyme-free biosensors. In non-enzymatic biosensors, polyacrylic acid hydrogel is integrated in a multifunctional matrix of graphene and lutetium phtalocyanine and used as a redox mediator (26). Zhu et al (27) described a novel nonenzymatic amperometric glucose sensor that was created using $\mathrm{Ni}$ nanoparticles homogeneously dispersed within and on the top of a vertically aligned CNT forest (CNT/Ni nanocomposite sensor), which was directly grown on a $\mathrm{Si} / \mathrm{SiO} 2$ substrate.

Other non-enzymatic sensors are of interest because of their higher sensitivity, selectivity, stability and are based on metal nanoparticles. Many materials are currently in research as enzyme free glucose sensors. Among these, perovskite oxides, such as strontium palladium perovskite, present increased catalytic activity, ionic and electronic conductivity after strontium A-site partial substitution by calcium ions (28).

Current research is orientated towards the use of microgels as glucose sensing systems. The changes of these microgels in terms of volume, fluorescence intensity and reflection or diffraction properties can be used. Starting with boronic acid, numerous types of gels have been developed. Li and Zhou (29) developed a method to manufacture sensors using a series of glucose-responsive microgels in which anionic dye molecules are incorporated and used as an optical technique of detection based on variation of fluorescence intensity, thus having the ability to convert physico-chemical changes into optical signals. Zhang et al (30) imagined a device using a PAMAM G1.0 microgel that provides a transdermal fluorescent signal.

The use of photoelectrochemical QD sensors can be combined with the enzymatic method allowing indirect glucose measurement. Tanne et al (31) reported the possibility of creating a photobioelectrochemical sensor using an indirect method, based on the $\mathrm{CdSe} / \mathrm{ZnS}$ ODs system.

New coatings for sensors have been created and can be made from organic or inorganic materials in order to increase their biocompatibility. The materials from which the coatings are made can be classified as follows: i) Natural such as alginate, chitin and chitosan; and ii) semisynthetic or synthetic derivatives; such as biodegradable polymers [poly(lactic-co-glycolic acid), polyethylene glycol, poly(2-hydroxyethyl methacrylate)] and polyvinyl alcohol $(19,32,33)$. Novel nanostructures with antimicrobial activity and good potential for increasing biocompatibility are currently being investigated (34).

Since enzymatic electrochemical sensors are the most studied and the most used sensors, the majority of commercially approved and available glucose biosensors, including Medtronic iPro2, Guardian RT, Guardian Connect, Dexcom G4 Platinum, G5, G6 and Abbot Freestyle Libre, use a quantitative amperometric assay and a wire enzyme technology. The sensors use a needle with a working electrode (Pt-Ir) with glucose-oxidase immobilized on the surface $(12,35)$. The needle is inserted in the subcutaneous tissue. The working electrode is covered with an inner layer, the enzyme layer and an outer diffusion limiting layer (11). Another $\mathrm{Ag} / \mathrm{AgCl}$ wire is wrapped around the working electrode and represents a counter electrode. Glucose reacts with glucose oxidase and a cofactor and is reduced to gluconolactone. The reduced enzyme is converted back into its oxidized form in the presence of the oxygen and hydrogen peroxide is produced. Electrochemical oxidation of the hydrogen peroxide in the reaction leads to the production of an electrical current signal (12-14,35). Following a process of calibration during which capillary glucose level is measured and entered by the patient or realized in the factory, the electric signal is transformed into a corresponding glucose value (12-14).

GlucoWatch Biographer uses another technology, which is a reverse iontophoresis that extract subcutaneous interstitial fluid through intact skin and measure glucose levels six times per $\mathrm{h}$ and up to $13 \mathrm{~h}$, using an electrode [https://www.accessdata.fda.gov/cdrh_docs/pdf/P990026S008b.pdf. Accessed Jun 2021]. Due to the time needed to obtain the sample and to measure glucose, the values displayed are $\sim 15 \mathrm{~min}$ behind the real determination [https://www.accessdata.fda. gov/cdrh_docs/pdf/P990026S008b.pdf. Accessed Jun 2021]. The technique does not puncture the skin, but due to site irritations, it was not largely used.

Senseonics Eversense CGM uses an optical, fluorescence-based technology to measure glucose levels in the interstitial fluid [https://www.medicaldevice-network. com/projects/senseonics-eversense-cgm-system. Accessed Jun 2021]. The sensor is coated with a fluorescent substrate that generates a small amount of light according to the interstitial glucose quantity. The signal is then converted into a glucose reading and transmitted via a mobile device such as smart phone or tablet to the patient [https://www.medicaldevice-network.com/projects/senseonics-eversense-cgm-system. Accessed Jun 2021].

CGMS can be also classified according to the modality used to place the sensor. The systems may be noninvasive, minimally invasive or invasive. Noninvasive in body methods to measure glucose comprise devices that do not puncture the skin and use transdermal sensors via optical analysis (13). Other noninvasive methods comprise external analysis of accessible body fluids like saliva or tears using spectroscopic methods. GlucoWatch Biographer was technically speaking a minimally invasive system and was clinically speaking not invasive [https://www.accessdata.fda. gov/cdrh_docs/pdf/P990026S008b.pdf. Accessed Jun 2021]. Needle-type continuous glucose sensors are minimally invasive. The sensor is made of a microneedle and a micropouch into which a very small amount of blood is withdrawn (13). The only recently approved invasive system is Senseonics' Eversense CGM. The sensor is inserted under the skin by the physician under local anesthesia, thus an incision is needed both for insertion and removal. Although the implantable sensor is only recently approved, previously attempts were made. A first study including 15 patients with type 1 diabetes and using a subcutaneously implanted, long-term, real-time glucose continuous glucose sensor in usual home setting was published in 2004 and reported an improved glucose control (36). 


\section{CGMS: In clinical practice}

As previously mentioned, at the beginning of CGMS use, the patient and the doctor were blinded for blood glucose levels. The systems needed to be calibrated 4 times per day with capillary blood glucose measurements. After three days, the data were downloaded into a computer and analyzed after values from SBGM were also entered. The new blinded system (iPro2 Recorder, Medtronic) is also called professional because it is intended to be used by physicians and can collect data up to 7 days (37). Specific software permits for data uploading and the physician can review and adjust the treatment and can thus identify specific patterns during the day or night. Since meal content and size influence the blood sugar levels (38), identifying some meal-related patters can help improving nutrition intervention. This system only completes and improves the classical SBGM but does not replace it. Conversely, the real-time CGMS (rtCGMS) transmits data continuously and has a wireless connection between the sensor and the device, thus allowing the patient to visualize glucose values and graphs (39). For the first time, real-time, personal use devices (Medtronic Guardian RT and Dexcom STS) had a lifetime of three days. The newest devices (Medtronic Guardian Connect, Dexcom G4 Platinum, Dexcom G5 and Dexcom G6) offer monitoring for 7-10 days. Since 2015, the devices are compatible with mobile phones. Certain features such as alarms for hypoglycemia or hyperglycemia can be programmed at individual targets, and glucose trends and rates of glucose variations are very useful for patients with type 1 diabetes (39) in preventing acute complications, but also in improving diabetes control on the long term (39). Furthermore, high glucose alerts allow the patient to adjust insulin doses, even in the context of other treatments affecting glycemia such as corticosteroids (40). Reports can be also generated. Numerous data are provided by sensors and are useful to assess the overall glucose control, the glucose variability or the periods spent in hypoglycemia or hyperglycemia (41). Capillary blood glucose measurements are required for calibration after inserting the sensor and 2-4 times per day afterward, depending on the model. The Dexcom G6 is factory calibrated and needs no finger puncture (42).

Intermittent glucose monitors (iCGMS), also known as flash glucose monitoring systems, have only two components, a mixed sensor/transmitter unit and a reader unit, and show glycemia values upon scanning the sensor (Abbot Free Style Libre). An $8 \mathrm{~h}$ trend is also provided, and reports for longer periods can be obtained. A very interesting advantage of iCGMS is that it is factory calibrated and does not need calibration for the whole period of 14 days of usage. It is also of lower cost. The limitations of this system are the following: i) The patient needs to scan the sensor with the reader device to have a blood glucose measurement; ii) in order not to lose accumulated data, a reading must be performed at least at $8 \mathrm{~h}$ interval; and iii) the lack of alarms for hypoglycemia and hyperglycemia makes this system inappropriate for patients with hypoglycemia unawareness. Although only the newest version of the device enables alarm notifications for low or high glucose level or trends for glucose changing (if the sensor is close to the reading device), the system is useful for rapid and repeated measurements of hemoglobin A1C (43). Another limitation is the lack of sharing features, but lately dedicated software might enable data sharing.

Senseonics Eversense CGM is the only skin implantable sensor. It comprises a smart transmitter, a rechargeable battery and a mobile application through which the patient receives and views the glycemic values, alerts and trends on his mobile device. It can be used up to 90 days, needs calibration every $12 \mathrm{~h}$ and has a good accuracy during hypoglycemic events.

Abbot Free Style Libre and Senseonics Eversense CGM are recommended for adults $>18$ years, while the Medtronic Guardian Connect system is indicated for patients aged 14-75 years. Medtronic Guardian Sensor 3 integrated with Medtronic Minimed 670G insulin pump are indicated for children $>7$ years (41) and Dexcom G5 and Dexcom G6 are approved for children $>2$ years (41).

CGMS presents some limitations. Issues about the bioanalytical performance, biocompatibility, length of wearing time, safety and costs are raised. Sensor accuracy is very important for compliance and crucial for clinical use. Interferences with other substances that may affect the enzyme-based reaction, stability of the sensor components, calibration and assessment methods are very important aspects. The sensors can interfere at the electrode potential with some other substances that are electroactive (12). Interferences with acetaminophen, ascorbic acid and uric acid have been described (12). To improve selectivity, permselective membranes may be taken into consideration and some polymeric materials (including cellulose acetate, Nafilon) and polyphenols were evaluated by Nichols et al (12). Free Style Libre and Dexcom G6 provide accurate readings even when interfering with acetaminophen (35). Fluorescence-based technology used by Eversense CGM is unaffected by ascorbic acid and acetaminophen. A recent animal study evaluated the sensor coating with a zwitterionic polymer and reported a significantly reduced noise signal and improvement in CGM performance and the need for frequent blood glucose recalibration (44). Another important aspect is the sensor biocompatibility, as also mentioned in other biomaterials used for different biological aims (45). As already shown in multiple studies, a significant increase in oxidative stress, pro-inflammatory status and endothelial dysfunction are seen in diabetic patients, especially when diabetes is associated with other cardiovascular risk factors or complicated with chronic renal disease at advanced stages (46-48).

\section{Conclusions}

In the last two decades, CGMS technologies introduced new ways to assess glucose control and have radically changed the management of type 1 diabetes, and improved glycemic control for certain categories of patients with type 2 diabetes. In addition, the devices are significantly improved for accuracy, biocompatibility, length of usage time, data collection, metrics, alarms and features sharing, allowing an individualized and improved treatment of diabetes.

\section{Acknowledgements}

Not applicable. 


\section{Funding}

No funding was received.

\section{Availability of data and materials}

Not applicable.

\section{Authors' contributions}

DAM, DSS, DS, GEB, IAV, TP, MODL, AEN, OS, DD, OGO designed the study, wrote the manuscript, performed a literature search and selected the studies. DAM, DSS, DS, GEB, IAV, TP, MODL, AEN, OS, DD, OGO critically revised the manuscript. All authors read and approved the final manuscript.

\section{Ethics approval and consent to participate}

Not applicable.

\section{Patient consent for publication}

Not applicable.

\section{Competing interests}

The authors declare that they have no competing interests.

\section{References}

1. International Diabetes Federation. IDF diabetes atlas, 9th edition. Brussels, Belgium: 2019. Available from: https://www.diabetesatlas.org.https://www.diabetesatlas.org/en/. Accessed Jun 2021.

2. Stroescu AE, Tanasescu MD, Diaconescu A, Raducu L, Constantin AM, Balan DG, Tarmure V and Ionescu D: Cardiovascular comorbidities, inflammation and serum albumin levels in a group of hemodialysis patients. Rev Chim 69: 926-929, 2018.

3. Stroescu AE, Tanasescu MD, Diaconescu A, Raducu L, Balan DG, Mihai A, Tanase M, Stanescu II and Ionescu D: Diabetic nephropathy: A concise assessment of the causes, risk factors and implications in diabetic patients. Rev Chim 69: 3118-3121, 2018.

4. Mandita A, Timofte D, Balcangiu-Stroescu AE, Balan D, Raducu L, Tanasescu MD, Diaconescu A, Dragos D, Cosconel CI, Stoicescu SM and Ionescu D: Treatment of high blood pressure in patients with chronic renal disease. Rev Chim 70: 993-995, 2019.

5. Timofte D, Mandita A, Balcangiu-Stroescu A, Balan D, Raducu L, Tanasescu MD, Diaconescu A, Dragos D, Cosconel CI, Stoicescu SM and Ionescu D: Hyperuricemia and cardiovascular diseases clinical and paraclinical correlations. Rev Chim 70 1045-1046, 2019.

6. Olaru OG, Constantin GI and Pena CM: Correlations of sialic acid with glycated hemoglobin A1c and glycemia in postmenopausal women with type-2 diabetes mellitus. Exp Ther Med 21: 286, 2021.

7. Clarke SF and Foster JR: A history of blood glucose meters and their role in self-monitoring of diabetes mellitus. $\mathrm{Br} \mathrm{J}$ Biomed Sci 69: 83-93, 2012.

8. Rock JA and Gerende LJ: Dextrostix method for determination of blood glucose levels. A statistical evaluation. JAMA 198: 231-236, 1966

9. No authors listed: Dextrometer for self-testing of blood glucose West J Med 136: 275, 1982.

10. Shichiri M, Kawamori R, Yamasaki Y, Hakui N and Abe H: Wearable artificial endocrine pancrease with needle-type glucose sensor. Lancet 2: 1129-1131, 1982.
11. Petrie JR, Peters AL, Bergenstal RM, Holl RW, Fleming GA and Heinemann L: Improving the clinical value and utility of CGM systems: Issues and recommendations: A joint statement of the European association for the study of diabetes and the American diabetes association diabetes technology working group. Diabetes Care 40: 1614-1621, 2017.

12. Nichols SP, Koh A, Storm WL, Shin JH and Schoenfisch MH: Biocompatible materials for continuous glucose monitoring devices. Chem Rev 113: 2528-2549, 2013.

13. Vaddiraju S, Burgess DJ, Tomazos I, Jain FC and Papadimitrakopoulos F: Technologies for continuous glucose monitoring: Current problems and future promises. J Diabetes Sci Technol 4: 1540-1562, 2010.

14. Olczuk D and Priefer R: A history of continuous glucose monitors (CGMs) in self-monitoring of diabetes mellitus. Diabetes Metab Syndr 12: 181-187, 2018.

15. Dovč K, Bratina N and Battelino T: A new horizon for glucose monitoring. Horm Res Paediatr 83: 149-156, 2015.

16. Aggidis AG, Newman JD and Aggidis GA: Investigating pipeline and state of the art blood glucose biosensors to formulate next steps. Biosens Bioelectron 15: 243-262, 2015.

17. Bailey T, Bode BW, Christiansen MP, Klaff LJ and Alva S: The performance and usability of a factory-calibrated flash glucose monitoring system. Diabetes Technol Ther 11: 787-794, 2015.

18. Limban C, Nuta DC, Missir AV, Roman R, Caproiu MT, Dumitrascu F, Pintilie L, Stefaniu A, Chifiriuc MC, Popa M, et al: Synthesis and characterization of new fluoro/trifluoromethyl-substituted acylthiourea derivatives with promising activity against planktonic and biofilm-embedded microbial cells. Processes 8: 503, 2020.

19. Chen C, Zhao XL, Li ZH, Zhu ZG, Qian SH and Flewitt AJ: Current and emerging technology for continuous glucose monitoring. Sensors (Basel) 17: 182, 2017.

20. Moon BU, de Vries MG, Cordeiro CA, Westerink BH and Verpoorte E: Microdialysis-coupled enzymatic microreactor for in vivo glucose monitoring in rats. Anal Chem 85: 10949-10955, 2013.

21. Mirea R, Ceatra L, Cucuruz AT, Ene R, Popescu E, Biris I and Cretu M: Advanced experimental investigation of used metallic biomaterials. Rom J Mater 59: 138-145, 2019.

22. Zhu Z, Garcia-Gancedo L, Flewitt AJ, Xie H, Moussy F and Milne WI: A critical review of glucose biosensors based on carbon nanomaterials: Carbon nanotubes and graphene. Sensors (Basel) 12: 5996-6022, 2012.

23. Zhu Z, Song W, Burugapalli K, Moussy F, Li YL and Zhong XH: Nano-yarn carbon nanotube fiber based enzymatic glucose biosensor. Nanotechnology 21: 165501, 2010.

24. Zhu ZG, Garcia-Gancedo L, Flewitt AJ, Moussy F, Li Y and Milne WI: Design of carbon nanotube fiber microelectrode for glucose biosensing. J Chem Technol Biotechnol 87: 256-262, 2012.

25. Zhang W, Du Y and Wang ML: On-chip highly sensitive saliva glucose sensing using multilayer films composed of single-walled carbon nanotubes, gold nanoparticles, and glucose oxidase. Sens Bio-Sens Res 4: 96-102, 2015.

26. Al-Sagura H, Komathia S, Khanb MA, Gurekc AG and Hassana A: A novel glucose sensor using lutetium phthalocyanine as redox mediator in reduced graphene oxide conducting polymer multifunctional hydrogel. Biosens Bioelectron 92: 638-645, 2017.

27. Zhu ZG, Garcia-Gancedo L, Chen C, Zhu XR, Xie HQ, Flewitt AJ and Milne WI: Enzyme-free glucose biosensor based on low density CNT forest grown directly on a $\mathrm{Si} / \mathrm{SiO} 2$ substrate. Sens Actuators B Chem 178: 586-592, 2013.

28. El-Ads EH, Galala A and Atta NF: The effect of A-site doping in a strontium palladium perovskite and its applications for non-enzymatic glucose sensing. RSC Adv 6: 16183-16196, 2016.

29. Li YY and Zhou SQ: A simple method to fabricate fluorescent glucose sensor based on dye-complexed microgels. Sens Actuators B Chem 177: 1363-1371, 2013.

30. Zhang X, Gao C, Lü S, Duan H, Jing N, Dong D, Shi C and Liu M: Anti-photobleaching flower-like microgels as optical nanobiosensors with high selectivity at physiological conditions for continuous glucose monitoring. J Mater Chem B 2: 5452-5460, 2014.

31. Tanne J, Schäfer D, Khalid W, Parak WJ, and Lisdat F: Light-controlled bioelectrochemical sensor based on CdSe/ZnS quantum dots. Anal Chem 83: 7778-7785, 2011.

32. Jayakumar R, Prabaharan M, Sudheesh Kumar PT, Nair SV and Tamura H: Biomaterials based on chitin and chitosan in wound dressing applications. Biotechnol Adv 29: 322-337, 2011. 
33. Morais JM,Papadimitrakopoulos F and Burgess DJ: Biomaterials/ tissue interactions: Possible solutions to overcome foreign body response. AAPS J 12: 188-196, 2010.

34. Vlad IM, Nuță DC, Ancuceanu RV, Caproiu MT, Dumitrascu F, Marinas IC, Chifiriuc MC, Măruţescu LG, Zarafu I, Papacocea IR, et al: New o-aryl-carbamoyl-oxymino-fluorene derivatives with MI-crobicidal and antibiofilm activity enhanced by combination with iron oxide nanoparticles. Molecules 26 3002, 2021.

35. Cappon G, Mettoretti M, Sparacino G and Facchinetti A: Continuous glucose monitoring sensors for diabetes management: A review of technologies and applications. Diabetes Metab J 43: 383-397, 2019.

36. Garg SK, Schwartz S and Edelman SV: Improved glucose excursions using an implantable real-time continuous glucose sensor in adults with type 1 diabetes. Diabetes Care 27: 734-738, 2004.

37. iPro2 User Guide. Medtronic MiniMed, Inc., 2016.

38. Balan DG, Stroescu AEB, Tanasescu MD, Diaconescu A, Raducu L, Mihai A, Tanase M, Stanescu II and Ionescu D: Nutritional intervention in patients with diabetic renal disease. A brief presentation. Rev Chim 69: 3178-3182, 2018.

39. Didyuk O, Econom N, Guardia A, Livingston K and Klueh U: Continuous glucose monitoring devices: Past, present, and future focus on the history and evolution of technological innovation. J Diabetes Sci Technol 15: 676-683, 2021.

40. Papacocea T, Popa E, Dana T and Papacocea R: The usefulness of dexamethasone in the treatment of chronic subdural hematomas. Farmacia 67: 140-145, 2019.

41. Danne T, Nimri R, Battelino T, Bergenstal RM, Close KL, DeVries JH, Garg S, Heinemann L, Hirsch I, Amiel SA, et al International consensus on use of continuous glucose monitoring. Diabetes Care 40: 1631-1640, 2017.

42. Wadwa RP, Laffel LM, Shah VN and Garg SK: Accuracy of a factory-calibrated, real-time continuous glucose monitoring system during 10 days of use in youth and adults with diabetes. Diabetes Technol Ther 20: 395-402, 2018.
43. Evans M, Welsh Z, Ells S and Seibold A: The impact of flash glucose monitoring on glycaemic control as measured by HbA1c: A meta-analysis of clinical trials and real-world observational studies. Diabetes Ther 11: 83-95, 2020.

44. Xie X, Doloff JC, Yesilyurt V, Sadraei A, McGarrigle JJ, Omami M, Veiseh O, Farah S, Isa D, Ghani S, et al: Reduction of measurement noise in a continuous glucose monitor by coating the sensor with a zwitterionic polymer. Nat Biomed Eng 2: 894-906, 2018.

45. Vrinceanu D, Bănică B, Cîrstoiu CF, Papacocea R and Papacocea T: 3D anatomically shaped titanium implant for the reconstruction of an orbital floor fracture with large posterior defect: A case report. Rom J Mater 48: 407-411, 2018

46. Timofte D, Ionescu D, Medrihan L, Mandita A, Rasina A and Damian L: Vascular calcification and bone disease in hemodialysis patient, assessment, association and risk factors. Nephrol Dial Transplant 22: 325-326, 2007.

47. Totan A, Balcangiu-Stroescu AE, Imre MM, Miricescu D, Balan D, Stanescu II, Ionescu D, Timofte D, Tanasescu MD and Greabu M: XOR-possible correlations with oxidative stress and inflammation markers in the context of diabetic kidney disease. Rev Chim 70: 1396-1398, 2019.

48. Timofte D, Dragos D, Balcangiu-Stroescu AE, Tănăsescu MD, Gabriela Bălan D, Răducu L, Tulin A, Stiru O and Ionescu D: Abdominal aortic calcification in predialysis patients: Contribution of traditional and uremia-related risk factors. Exp Ther Med 20: 97-102, 2020. 